\title{
Differential stability of mitochondrial mRNA in HeLa cells
}

\author{
Janusz Piechota ${ }^{1}$, Rafał Tomecki ${ }^{1}$, Kamil Gewartowski ${ }^{2}$, Roman Szczęsny ${ }^{1,2}$, \\ Aleksandra Dmochowska ${ }^{1,2}$, Marek Kudła ${ }^{1}$, Lien Dybczyńska ${ }^{1}$, Piotr P. Stepien ${ }^{1,2}$ \\ and Ewa Bartnik ${ }^{1,2 凶}$ \\ ${ }^{1}$ Department of Genetics, Warsaw University and ${ }^{2}$ Institute of Biochemistry and Biophysics, Polish Academy of \\ Sciences, Warszawa, Poland; ${ }_{e}$-mail: ebartnik@ibb.waw.pl
}

Received: 30 August, 2005; revised: 17 October 2005; accepted: 19 October 2005 available on-line: 21 December, 2005

\begin{abstract}
The physiological significance and metabolism of oligoadenylated and polyadenylated human mitochondrial mRNAs are not known to date. After study of eight mitochondrial transcripts (ND1, ND2, ND3, ND5, CO1, CO2, ATP6/8 and Cyt. b) we found a direct correlation between the halflives of mitochondrial mRNAs and their steady-state levels. Investigation of the mt-mRNA decay after thiamphenicol treatment indicated that three transcripts (ND2, ND3 and Cyt. b) are significantly stabilized after inhibition of mitochondrial translation. Careful analysis one of them, ND3, showed that inaccurate processing of the H-strand RNA precursor may occasionally occur between the ND3 and tRNA ${ }^{\text {Arg }}$ locus leading to synthesis of ND3 mRNAs lacking the STOP codon. However, analysis of the oligo(A) fraction observed in case of the ND3 indicates that partially polyadenylated mRNAs are linked rather to the transcription process than to the translation-dependent deadenylation. Analysis of ND3 mRNA turnover in cells with siRNA-mediated knock-down of the mitochondrial poly(A) polymerase shows that strongly decreased polyadenylation does not markedly affect the decay of this transcript. We present a model where oligoadenylated mitochondrial transcripts are precursors of molecules containing full length poly(A) tails.
\end{abstract}

Keywords: polyadenylation, poly(A) polymerase, HeLa, mitochondria, mRNA turnover

In human mitochondria both the $\mathrm{H}$ and $\mathrm{L}$ DNA strands are transcribed and processed into smaller units corresponding to particular mRNAs, tRNAs and rRNAs (for review see: Ojala et al., 1981, and Taanman, 1999).

After their release from the RNA precursors, mitochondrial mRNAs are polyadenylated; the length of $\operatorname{poly}(\mathrm{A})$ tails was estimated with the use of biochemical methods to be around 50-80 nucleotides (Perlman et al., 1973). However, the role of polyadenylation of the mitochondrial transcripts in cells from humans and other mammals has not been fully characterized yet.

The influence of polyadenylation on mRNA metabolism depends on the group of organisms and the cellular compartment in which this process takes place. In eukaryotes addition of a poly(A) tail to mRNA protects transcripts against rapid degra- dation (Mitchell \& Tollervey, 2000). In bacteria (Xu \& Cohen, 1995), plant mitochondria (Gagliardi \& Leaver, 1999) and chloroplasts (Hayes et al., 1999) addition of the poly(A) tails redirects transcripts to degradation. Moreover, polyadenylation is required for degradation of rRNA and snoRNA precursors in the nucleus in yeast (LaCava et al., 2005).

Recently, the gene coding for the human mitochondrial poly(A) polymerase (hmtPAP) was identified by our group (Tomecki et al., 2004). Silencing of its expression by RNA interference resulted in accumulation of oligoadenylated mitochondrial mRNA transcripts with about 5 adenosine residues at the $3^{\prime}$ terminus, and the steady-state levels of such mt-mRNAs were even higher following the inhibition of hmtPAP expression in comparison with untreated cells. This suggested to us that non-polyadenylated transcripts

Abbreviations: ATP 6/8, ATP synthase subunit 6 and 8; DMEM, Dulbecco's modified Eagle's medium; hmtPAP, human mitochondrial poly(A) polymerase; $\mathrm{mt}$, mitochondrial; PAP, poly(A) polymerase; RNAi, RNA interference; rRNA, ribosomal RNA; siRNA, small interfering RNA; snoRNA, small nuclear RNA. 
are relatively stable in human mitochondria. In contrast to this, Nagaike et al. (2005), who described hmtPAP independently, observed decreased levels of several, but not all mt-mRNAs after hmtPAP silencing, thus they hypothesized that shortening of poly(A) tails of mitochondrial transcripts enhances their degradation. In view of these discrepancies the role of mitochondrial polyadenylation in controlling mt-mRNA stability still remains controversial.

The mechanism of RNA degradation in human mitochondria is largely unknown. Temperley et al. (2003) described a patient harboring a microdeletion $\mu \Delta 9025$, resulting in removal of the translation termination codon of the ATP6/8 transcript. After analysis of the cell line isolated from the patient they suggested a mechanism removing defective transcripts in human mitochondria that would involve translation-dependent deadenylation and subsequent degradation of ATP6/8 transcripts lacking the STOP codon. Jesina et al. (2004) analyzing the same mutation in a different patient suggested that polyadenylation was not impaired and did not affect transcript processing.

The origin and fate of the oligoadenylated mt-mRNA fraction is unclear and requires further investigations. These should include accurate measurements of half-lives and levels of individual mRNAs, as well as their oligo- and polyadenylated fractions. In this paper we have examined the turnover of mitochondrial transcripts in normal HeLa cells and cells with affected mitochondrial translation. Furthermore, we analysed metabolism of the polyadenylated and oligoadenylated ND3 transcripts as well as the interplay between those two fractions.

\section{MATERIALS AND METHODS}

Materials and cell cultures. Tissue culture media, supplements, fetal calf serum and thiamphenicol were from Sigma. Plasticware was from Sarstedt. Actinomycin D was purchased from Calbiochem. $\left[\alpha{ }^{32} \mathrm{P}\right]$ ATP $\left(3000 \mathrm{Ci} \cdot \mathrm{mmol}^{-1}\right)$ and $\left[\gamma^{-32}{ }^{32}\right]$ ATP $(4500$ $\mathrm{Ci} \cdot \mathrm{mmol}^{-1}$ ) were from MB Biochemicals. Enzymes were obtained from Fermentas.

The HeLa cell line was used in all experiments. Cells were grown in DMEM medium supplemented with $10 \%$ fetal bovine serum, $2 \mathrm{mM}$ glutamine, $1 \mathrm{mM}$ pyruvate and $50 \mu \mathrm{g} / \mathrm{ml}$ uridine, in conditions of $37^{\circ} \mathrm{C}, 5 \% \mathrm{CO}_{2}$ and $100 \%$ humidity. OPTIMEM (Gibco) medium was employed in RNAi experiments, according to manufacturer's instructions.

Northern analysis. RNA was isolated using TRI Reagent (Sigma) according to the manufacturer's instructions. Aliquots of 4-10 $\mu \mathrm{g}$ RNA were electrophoresed in $1 \%$ denaturing formaldehyde/agarose or $5 \%$ polyacrylamide/urea sequencing gels (Sambrook
\& Russel, 2001) and transferred to Hybond-N filter (Amersham International), following the manufacturer's instructions. Filters were prehybridized in PerfectHyb ${ }^{\mathrm{TM}}$ Plus hybridization buffer (Sigma). PCR products enclosing the following mtDNA fragments were used as probes: 1756-2444 (16S rRNA), 35364239 (ND1), 4832-5570 (ND2), 8648-9199 (ATP6/8), 9911-10469 (ND3), 9181-9867 (CO3), 10985-11708 (ND4/4L), 14227-15419 (Cyt. b). PCR product used as 7SL RNA probe was amplified with primers 7SL-F: TCGGGTGTCCGCACTAAGTT and 7SL-R: TGGCTATTCACAGGCGCGAT. The PCR product was labeled using Hexa- or Deca-Label DNA Labeling Kit (Fermentas), according to the manufacturer's instructions.

Poly(A) tail sequence and length analysis of the ND3 transcript. The analysis of poly(A) tail nucleotide composition was performed essentially as described in (Tomecki et al., 2004). The poly(A) tail length was assayed as follows: the PCR products obtained after reamplification were electrophoresed in denaturing acrylamide gels with formamide (Litt et al., 1993) and visualized using Silver Stain Kit (Kucharczyk, Poland). The predicted length of the PCR reamplification product without poly(A) is 155 bp.

RNAi experiments. RNA interference against $h m t P A P$ was performed exactly in the same manner as described elsewhere (Tomecki et al., 2004). Silencing procedure was carried out simultaneously using four independent plates. Seventy two hours after siRNA-mediated silencing cells were subjected to the time-course of actinomycin D treatment. Four other plates treated in the same way except for siRNA addition served as a control.

Decay rate of the mitochondrial transcripts. Decay rates of the mitochondrial transcripts were measured for control (untreated) HeLa cells, HeLa cells treated with thiamphenicol $(50 \mu \mathrm{g} / \mathrm{ml})$ for three days and HeLa cells with silenced hmtPAP. Control and thiamphenicol-treated HeLa cells were seeded into plates at initial density of $3 \times 10^{4} / \mathrm{cm}^{2}$ and cultured three days in DMEM medium alone or with addition of the drug.

Actinomycin D was added at the concentration of $5 \mu \mathrm{g} / \mathrm{ml}$ to three plates in each experiment. Cells were collected in one-hour intervals and suspended in TRI reagent (Sigma). RNA was isolated according to the manufacturer's protocol. RNA isolated from the fourth plate (prior to the actinomycin $\mathrm{D}$ treatment) served as a control $(0 \mathrm{~h}$ time point). In the case of thiamphenicol-treated cells two inhibitors (actinomycin D and thiamphenicol) were present during decay experiments. Steady-state levels of transcripts were measured using the Northern-blot analysis and the real-time PCR technique. The halflives of the transcripts were calculated as described 
in (Chrzanowska-Lightowlers et al., 1994). In short: semi-log plots of steady-state level versus time were plotted. Then the parameters of the linear equation were calculated using the best fit algorithm. Then, the steady-state levels of the transcripts at 0 time point were calculated from the parameters of the linear equation. Data are presented as estimation of the steady-state level \pm standard deviation of the estimation. The half-life times were calculated from the slope of the equation. The standard deviations of the half-lives correspond to standard deviation of the slope estimation.

Real-time PCR analysis. DNA-free RNA samples were prepared using deoxyribonuclease I (Fermentas) according to the manufacturer's instructions. Then reverse transcription was performed using Omniscript RT Kit (Qiagen) and oligo(dT) ${ }_{18}$ primer. Real-time PCR reaction was performed on LightCycler (Roche) using QuantiTect SYBR Green PCR Kit (Qiagen). Primers specified in Table 1 were employed for the relative quantification of the levels of mitochondrial transcripts, while those described by Vandesompele et al. (2002) were used for standardization.

\section{RESULTS}

Turnover of the polyadenylated mitochondrial mRNA transcripts in HeLa cells

To investigate human mtRNA half-lives and stability we used a non-radioactive technique based on the inhibition of transcription and subsequent measurement of the steady-state levels of the mitochondrial mRNA transcripts in serial time points by reverse transcription with oligo(dT) primer, followed by real-time PCR analysis. The analysis was performed for eight transcripts: ND1, ND2, CO1, CO2, ATP6/8, ND3, ND5 and Cyt. b. This approach allowed us to study not only the half-lives of the individual transcripts, but also their abundance in relation to each other. The results are presented in Table 2. The ATP6/8 transcript was analyzed along with its precursor, comprising the ATP6/8 and $\mathrm{CO} 3$ mRNAs (RNA14/15 in ref. 17), which had been previously shown to be polyadenylated (Jesina et al., 2004). From the Northern-blot analysis we estimated that the ratio of ATP6/8 transcript to its precursor is about 4:1 in untreated HeLa cells and 10:1 in HeLa cells treated with thiamphenicol (not shown), thus the majority of the signal observed in the real-time PCR reaction was derived from the mature transcript.

The eight analyzed transcripts can be divided into three different groups according to their steadystate levels and half-lives. The most numerous group consists of ND1, ND2, CO1, ND5 and Cyt. b mRNAs with half-lives between 2 and $3 \mathrm{~h}$. The second class includes only one transcript, ND3, which displays a relatively short half-life of about one hour, correlated with a lower relative steady-state level. Finally, the half-lives of the CO2 and ATP6/8 transcripts that constitute the third group, are longer than those observed for the first group, which correlates with a 2-fold increase of their steady-state levels. These results show that decay rate after excision from the precursor is an important step determining the abundance of the mRNA species encoded by the $\mathrm{H}$ strand of the mtDNA.

\section{Influence of the mitochondrial translation on the decay of the mt-mRNAs}

Van Hoof et al. (2002) and Frischmeyer et al. (2002) reported a surveillance mechanism present in eucaryotic cytoplasm, which removes transcripts without STOP codon in a degradation process, which is strongly dependent on the translation of such aberrant transcripts. Recently such translation-dependent degradation process of aberrant transcripts was reported to be present in human mitochondria isolated from a patient with microdeletion removing the STOP codon from the ATP6/8 transcript (Temperley

Table 1. Primers used in real-time PCR reaction for quantification of the levels of the mitochondrial transcripts.

\begin{tabular}{lll}
\hline Transcript & Forward primer & Reverse primer \\
\hline ND1 & ACACTAGCAGAGACCAACCGAA & GGGAGAGTGCGTCATATGTTGT \\
ND2 & CTATCTCGCACCTGAAACAAGC & GGTGGAGTAGATTAGGCGTAGG \\
CO1 & TACGTTGTAGCCCACTTCCACT & GGATAGGCCGAGAAAGTGTTGT \\
CO2 & GTAGTACTCCCGATTGAAGCCC & ATTCTAGGACGATGGGCATGAA \\
ATP6/8 & CCATCAGCCTACTCATTCAACC & GCGACAGCGATTTCTAGGATAG \\
ND3 & CCACAACTCAACGGCTACATAG & CACTCATAGGCCAGACTTAGGG \\
ND5 & TCTACCCTAGCATCACACACCG & GTTGAGGTGATGATGGAGGTGG \\
Cyt. b & TATTCGCCTACACAATTCTCCG & GCTTACTGGTTGTCCTCCGATT \\
\hline
\end{tabular}


et al., 2003). We were interested whether translation plays an important role in degradation of mitochodrial polyadenylated transcripts in normal cells. To answer this question we analyzed the half-lives of selected transcripts in mitochondria with blocked translation by pre-treatment of the cells with thiamphenicol for three days. The results compared with those obtained for untreated cells are summarized in Table 2.

Two distinct groups of transcripts were found. The decay ratios for treated/untreated cells are around 1 for ND1, CO1, CO2 and ATP6/8 transcripts, indicating that their degradation is not strongly affected by lack of translation. A somewhat different group of transcripts was analyzed by Chrzanowska-Lightowlers et al. (1994), nonetheless their results for CO1, CO2 and ATP6/8 are similar to ours. However, three other transcripts (ND2, ND3 and Cyt. b) are significantly more stable after inhibition of mitochondrial translation. Therefore, we can conclude that the rate of degradation, for some of the mitochondrial transcripts, is affected by translation inhibition in normal HeLa cells. Interestingly in all three transcripts only the first nucleotide of the STOP codon is encoded in the mtDNA. The other two nucleotides are added by the polyadenylation process, leading to formation of the UAA STOP codon.

\section{Analysis of the $3^{\prime}$ ends of the ND3 transcript}

Translation-dependent mRNA degradation appears to be linked to the aberrant transcripts, lacking STOP codons (van Hoof et al., 2002, Frisch- meyer et al., 2002; Temperley et al., 2003). Thus stabilization of ND2, ND3 and Cyt. b transcripts after thiamphenicol treatment may suggest that at least some of the synthesized molecules are defective at their $3^{\prime}$ ends and are subsequently degraded in a translation-dependent degradation pathway. Aberrant mRNAs could be formed by inaccurate excision of the transcript from the RNA precursor. Therefore, we decided to check the $3^{\prime}$ end of the transcripts for the presence of molecules without the STOP codon. We chose the ND3 transcript for further analysis. This transcript is stabilized by thiamphenicol treatment, possesses the shortest half-life and displays the lowest steady-state level. Moreover, we were able to observe a distinct oligoadenylated fraction only for this mRNA (Tomecki et al., 2004). Such oligoadenylated RNAs were suggested to be the result of the deadenylation of transcripts lacking the STOP codon (Temperley et al., 2003). If the increased stability of the ND3 transcript after thiamphenicol treatment was indeed due to the lack of translation-associated degradation of aberrant transcripts, STOP codon lacking ND3 transcripts should be still present among the polyadenylated molecules in cells treated with thiamphenicol. Moreover, in control cells the oligoadenylated fraction should be composed of transcripts without the STOP codon (which underwent translation-dependent deadenylation according to model proposed by Temperley et al. (2003)) but the polyadenylated fraction should not contain any such species.

In order to analyze the $3^{\prime}$ ends of the ND3 molecules we performed circularization of the mRNA and sequencing essentially as described in

Table 2. The steady-state levels and decay rates of the mitochondrial polyadenylated transcripts.

The steady-state levels and half-life times of the transcripts in untreated and thiamphenicol treated HeLa cells were estimated by reverse transcription of the total RNA with oligo $\left(\mathrm{dT}_{18}\right)$ followed by real-time PCR analysis (for details see Materials and Methods). For better visualisation of the results, the steady-state levels were recalculated taking the ND1 level as 100. The half-lives were estimated in an experiment with actinomycin D, an inhibitor of transcription. RNA samples from successive time points were collected and measured as detailed in the Materials and Methods section. Thiamphenicol treated cells used in the decay experiments were pre-treated with the inhibitor for three days. Steady-state levels are presented as the value \pm confidence level, whereas half-life times are represented as the value \pm standard deviation.

\begin{tabular}{lccccc}
\hline Transcript & Untreated cells & \multicolumn{2}{c}{ Thiamphenicol treated cells } & \multicolumn{2}{c}{ STOP codon } \\
\cline { 2 - 5 } & Steady-state level & Half-life time (min) & Half-life time (min) & Relative change \\
\hline ND1 & $100 \pm 4$ & $219 \pm 22$ & $273 \pm 21$ & 1.25 & TA \\
ND2 & $91 \pm 11$ & $142 \pm 3$ & $296 \pm 22$ & 2.09 & T \\
CO1 & $97 \pm 19$ & $204 \pm 91$ & $236 \pm 65$ & 1.15 & AGA \\
CO2 & $234 \pm 19$ & $297 \pm 97$ & $277 \pm 78$ & 0.94 & TAG \\
ATP6/8 & $177 \pm 69$ & $424 \pm 104$ & $506 \pm 51$ & 1.19 & TAA \\
ND3 & $28 \pm 1$ & $59 \pm 1$ & $132 \pm 16$ & 2.23 & T \\
ND5 & $102 \pm 17$ & $120 \pm 27$ & & & TAA \\
Cyt. B & $139 \pm 16$ & $132 \pm 24$ & $406 \pm 27$ & 3.06 & T \\
\hline
\end{tabular}


our previous work (Tomecki et al., 2004). We sequenced the cloned cDNAs obtained from the polyadenylated fraction of thiamphenicol-treated HeLa cells, as well as poly(A) and oligo(A) fractions isolated from untreated HeLa cells. As shown in Table 3 we found ND3 transcripts without a STOP codon among the polyadenylated fraction from cells treated with thiamphenicol. Such aberrant molecules were not found in the polyadenylated fraction from normal cells. mRNAs lacking the STOP codon were also observed among oligoadenylated transcripts from normal cells, but surprisingly we found only four aberrant cDNAs among 19 sequenced clones. The majority of the sequenced clones possessed proper $3^{\prime}$ ends. This result should not be expected if the oligoadenylated fraction originated exclusively from the translation-dependent degradation of polyadenylated mt-mRNAs, according to the model proposed by Temperley et al. (2003) for the ATP6/8 transcript.

The calculated average length of the poly(A) tails in normal cells is 46 nucleotides. The poly(A) tails of the transcripts isolated from thiamphenicoltreated cells are slightly longer (51 nucleotides on average) Although the difference is only 5 nucleotides, it appears to be statistically significant ( $t$-test, $P=0.0038)$. The average length of the sequenced
oligo(A) tails was 10 nucleotides. The lengths of the extensions found among sequenced cDNA clones varied between 1 and 28 nucleotide (Table 3). All sequenced poly(A) and oligo(A) tails are composed almost exclusively of adenine nucleotides. Among 1608 sequenced nucleotides we found three guanines. This result confirms and extends the sequencing data described previously (Tomecki et al., 2004).

\section{Oligoadenylated ND3 transcripts in normal HeLa cells do not originate from a translation-dependent decay pathway}

In order to analyze the origin and fate of the oligoadenylated ND3 mRNAs in the cells with arrested protein synthesis we examined the effects of translation inhibition during treatment of HeLa cells with thiamphenicol for 6 days. We analyzed the presence of the polyadenylated and oligoadenylated fractions of the ND3 transcript in 2-day intervals.

Northern-blot analysis indicated that the steady-state level of the poly(A) fraction of the ND3 mRNA is increased (about 1.5 fold) after thiamphenicol treatment (Fig. 1A). Trying to explain the possible cause of this increase, we performed high resolution Northern-blot analysis for the same samples. The results presented in Fig. 1B clearly show that

Table 3. Analysis of polyadenylated ND3 transcripts from cells treated with thiamphenicol.

RNA was isolated from normal cells or cells pre-treated with thiamphenicol, circularized and, after reverse transcription, used in the PCR reaction. PCR products were electrophoresed in $2 \%$ agarose gels. Bands with lengths corresponding to the polyadenylated or oligoadenylated fractions were excised from the gel and cloned. Plasmids isolated from single colonies were used as templates in nested PCR. The obtained PCR products were sequenced.

\begin{tabular}{|c|c|c|c|c|c|}
\hline \multicolumn{2}{|c|}{ Poly(A) - control cells } & \multicolumn{2}{|c|}{ Poly(A) - thiamphenicol } & \multicolumn{2}{|c|}{ Oligo(A) - control cells } \\
\hline Sequence & Quantity & Sequence & Quantity & Sequence & Quantity \\
\hline $\operatorname{GAAT}(\mathbf{A})_{41}$ & $4 \times$ & GAAT $(\mathbf{A})_{43}$ & $1 \times$ & GAAT $(\mathbf{A})_{1}$ & $1 \times$ \\
\hline GAAT $(\mathbf{A})_{42}$ & $3 \times$ & GAAT $(\mathbf{A})_{46}$ & $1 \times$ & GAAT $(\mathrm{A})_{4}$ & $1 \times$ \\
\hline GAAT $(\mathbf{A})_{44}$ & $1 \times$ & GAAT $(\text { A })_{51}$ & $1 \times$ & GAAT (A) ${ }_{6}$ & $2 \times$ \\
\hline GAAT $(A)_{9} G(A)_{36}$ & $1 \times$ & GAAT $(\mathbf{A})_{52}$ & $2 \times$ & GAAT (A) ${ }_{9}$ & $1 \times$ \\
\hline GAAT $(\mathbf{A})_{47}$ & $1 \times$ & GAAT $(\mathbf{A})_{53}$ & $4 \times$ & GAAT $(\text { A })_{10}$ & $3 x$ \\
\hline GAAT $(\mathbf{A})_{49}$ & $1 \times$ & GAAT $(\text { A })_{54}$ & $2 \times$ & GAAT $(\text { A })_{11}$ & $1 \times$ \\
\hline GAAT $(\mathbf{A})_{53}$ & $2 \times$ & GAAT $(\mathbf{A})_{56}$ & $1 \times$ & GAAT $(\mathbf{A})_{12}$ & $2 \times$ \\
\hline \multirow[t]{7}{*}{ GAAT $(\mathbf{A})_{57}$} & $1 \times$ & GAAT $(\mathbf{A})_{57}$ & $1 \times$ & GAAT $(\mathbf{A})_{13}$ & $1 \times$ \\
\hline & & GAA $\quad(A)_{46}$ & $1 \times$ & GAAT $(\mathbf{A})_{15}$ & $1 \times$ \\
\hline & & GAA $\quad(\mathrm{A})_{47}$ & $1 \times$ & GAAT $(\text { (A) })_{19}$ & $1 \times$ \\
\hline & & & & GAAT $(\mathrm{A})_{\mathbf{1 4}}(\mathrm{G})_{\mathbf{2}}(\mathrm{A})_{12}$ & $1 \times$ \\
\hline & & & & GAA $\quad(A)_{1}$ & $1 \times$ \\
\hline & & & & GAA $\quad(A)_{10}$ & $2 \times$ \\
\hline & & & & GAA $\quad(A)_{12}$ & $1 \times$ \\
\hline
\end{tabular}


the steady-state level of the oligoadenylated fraction is unchanged after thiamphenicol treatment. Thus, the increased steady-state level of the ND3 transcript presented in Fig. 1A is exclusively due to specific increase of the polyadenylated fraction observed in Fig. 1B. This indicates that the translational inhibition does not affect the level of the oligoadenylated fraction, but specifically stabilizes the polyadenylated trancripts. We thus suggest that oligoadenylated ND3 transcripts, at least in the HeLa cell line, do not seem to be the result of translation-dependent degradation.

\section{Oligoadenylated ND3 transcript levels are affected by the lack of mitochondrial transcription}

If oligoadenylated ND3 transcripts are not due to translation-dependent degradation, they could be formed by adenylation of newly formed transcripts. We therefore analyzed the presence of the poly(A) and oligo(A) fractions after inhibition of mitochondrial transcription by actinomycin D. A rapid decrease of the oligo(A) fraction level was observed (Fig. 2A), so that virtually no oligoadenylated transcripts could be detected after one hour of actinomycin $\mathrm{D}$ treatment by high resolution Northernblot or PCR-based poly(A) tail length analysis. As mentioned above and shown in Fig. 2B, the oligo(A) fraction is still observed in cells pre-treated with thiamphenicol. However, when actinomycin D is added to these cells, the oligoadenylated ND3 mRNA also rapidly disappears.

These data suggest that the appearance of the oligoadenylated fraction observed in the case of ND3 transcript is linked to ongoing transcription. However, the fate of the oligoadenylated transcripts is still unclear. They could be either an intermediate between the "naked" molecule excised from the precursor and the fully polyadenylated mature transcript or an intermediate product of polyadenylated transcript degradation. Thus, the rapid disappearance of the oligo(A) fraction could be explained by their elongation to polyadenylated transcripts or conversely, by their rapid decay. Another possibility is that these two fractions are entirely independent.

\section{Degradation of the ND3 transcript in cells after depletion of mitochondrial poly(A) polymerase}

Results obtained above indicate that oligoadenylated ND3 mRNAs more likely originate from the transcription than from translation-dependent deadenylation and their turnover is relatively fast. However, it remains unknown whether oligoadenylated transcripts are rapidly degraded or rather serve as intermediates of the polyadenylation. As has been stated above, in control cells oligoadenylated species

A

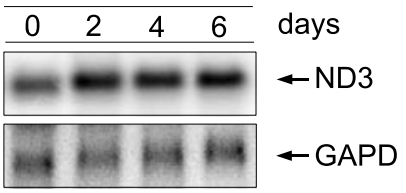

B

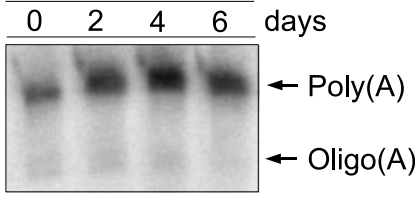

Figure 1. Changes in the steady-state levels of the ND3 poly(A) and oligo(A) fractions after thiamphenicol treatment of HeLa cells.

HeLa cells were treated with thiamphenicol for 6 days. Cells were collected in 2-day intervals and analysed using Northern blot (A) and high resolution Northern blot (B). Northern blot was standardized for the glyceraldehyde-3phosphate dehydrogenase gene (GAPD).

constitute only a tiny fraction of the given messenger, so it is difficult to analyze their stability under normal conditions. However, we took advantage of the fact that the oligoadenylated fraction accumulates to a considerable level in cells treated with siRNAs against hmtPAP, especially in the case of ND3 mRNA. This enabled us to analyze the decay of oligoadenylated ND3 transcripts more precisely. We examined the fate of both polyadenylated and oligoadenylated fractions of ND3 mRNA in mocktreated and siRNA-treated cells by performing high resolution Northern-blot analysis.

Basing on the high resolution Northern-blot data (Fig. 3) we first calculated the half-life of the ND3 transcript in mock-treated cells and siRNAtreated cells (Table 4). Calculated half-lives of the total ND3 transcripts (combined oligo(A) and poly(A)

A
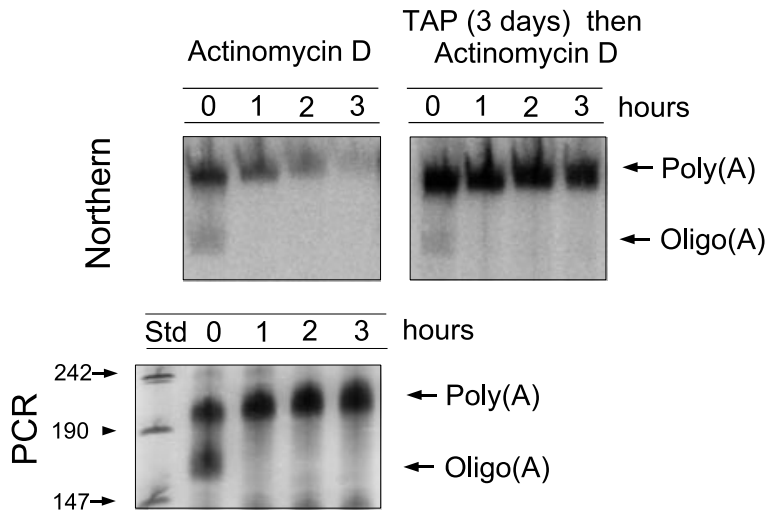

Figure 2. Relation between the oligo(A) fraction and the mitochondrial transcription.

Normal (A) and thiamphenicol pre-treated (B) HeLa cells were treated with actinomycin $\mathrm{D}$ and analysed using high resolution Northern blot and, in case of untreated cells, PCR-based techniques. 
A

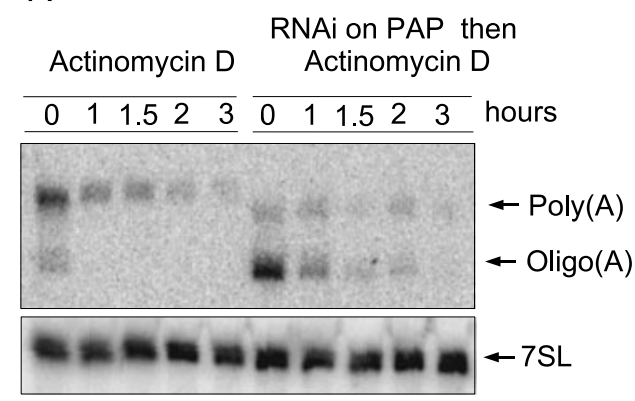

B

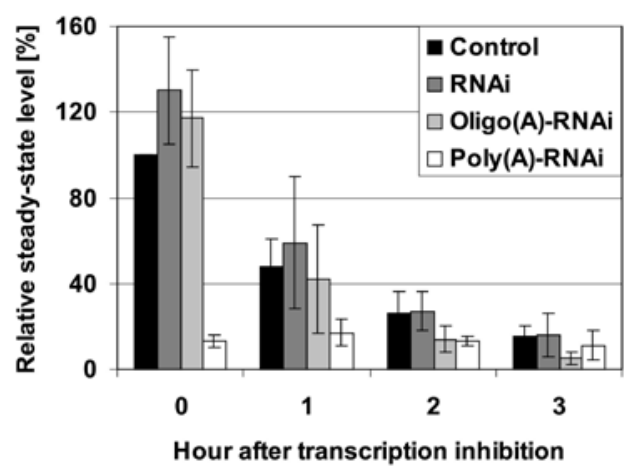

Figure 3. Decay of the ND3 in normal cells and cells with disrupted expression of the mitochondrial poly(A) polymerase.

Cells with disrupted expression of the hmtPAP were prepared by RNAi technique as previously described (Tomecki et al., 2004). Control cells were prepared in the same manner except addition of the appropriate oligoribonucleotides. Prepared cells were assayed for decay experiment as described in Materials and Methods section. RNA samples were analysed with high resolution Northern blot technique; (A) exemplary result of the hybridization to ND3 transcript. 7SL RNA was used for standardization; (B) chart presenting the decay of the oligo(A) and poly(A) fractions in normal and RNAi-treated cells. Presented results were obtained from 3 independent experiments. For better visualisation of the results, the steady-state levels were recalculated taking the level of the poly(A) fraction in mock-treated cells as 100 .

fractions) are almost the same for normal cells and cells with disrupted hmtPAP $(66 \pm 2$ and $59 \pm 3 \mathrm{~min}$, respectively). The difference of $7 \mathrm{~min}$ is not statistically significant $(P>0.05)$. The poly(A):oligo(A) ratio in normal cells is close to $10: 1$. In contrast, in cells with disrupted mitochondrial poly(A) polymerase the ratio is partially inverted (around 1:4) (Tomecki et al., 2004). Almost identical half-lives of the total ND3 transcripts in both cases indicate that the lack of polyadenylation of the ND3 transcripts has no significant effect on their stability.

The calculated half-life of the most abundant ND3 transcript fraction in siRNA-treated cells, i.e. oligoadenylated mRNAs was $39.4 \pm 0.2 \mathrm{~min}$, significantly less than the half-life of the overall ND3 transcript $(59 \pm 3)$. This result alone could suggest that the oligoadenylated mRNAs are indeed less
Table 4. Calculated half-lives of various ND3 fractions in normal cells and cells with disrupted expression of the mitochondrial poly(A) polymerase.

Data obtained from analysis of the Northern blot depicted on Fig. 3 were used to calculate the half-lives the oligo(A) and poly(A) fractions. The steady-state level of the polyadenylated ND3 transcripts appears to be stable during the analysed period of $3 \mathrm{~h}$. The oligo(A) fraction in the normal cells disappears during first $30 \mathrm{~min}$. Additionally, signals from the oligo(A) and poly(A) fractions were added in order to obtain total amounts of the ND3 transcript in successive time points. The summarized signals were used to calculate the half-life of the total amount of the ND3 mRNAs (oligo(A) + poly(A)) in the control cells and cells with disrupted expression of the mitochondrial poly(A) polymerase. The values are given a number \pm standard deviation.

\begin{tabular}{ll}
\hline Analysed fraction & Half-life (min) \\
\hline RNAi: oligo(A) & $39.4 \pm 0.2$ \\
Control: poly(A) + oligo(A) & $66 \pm 2$ \\
RNAi: poly(A) + oligo(A) & $59 \pm 3$ \\
\hline
\end{tabular}

stable than the polyadenylated transcripts. Surprisingly, we noticed that the steady-state level of the poly(A) fraction is relatively stable after inhibition of transcription in cells with silenced hmtPAP. There is no obvious reason why the polyadenylated ND3 transcripts are not degraded. Thus, the explanation of the stable steady-state level is that the degraded transcripts from the poly(A) fraction are replaced by newly synthesized ones. The only source of the novel polyadenylated transcripts is the oligo(A) fraction. Thus, faster disappearance of the oligo(A) fraction in RNAi treated cells possibly is not due to its faster degradation, but polyadenylation to fully polyadenylated mRNAs.

\section{hmtPAP knock down influences the mt-mRNA steady-state levels in a differentiated manner}

As has been mentioned previously, some inconsistency can be seen between the data reported by us (Tomecki et al., 2004) and the results obtained by Nagaike et al. (2005), concerning the outcome of siRNA-mediated silencing of hmtPAP expression on the steady-state levels of mitochondrial RNAs. In the meantime, we managed to address this issue more accurately, as well as to extend our parallel investigation to a greater number of mitochondrial transcripts. Results of one representative experiment are shown in Fig. 4, and the quantitative analysis is presented in Table 5. We observed a significant increase of the transcripts coding for several NADH dehydrogenase subunits, namely ND1, ND2, ND3 and to a lesser extent also ND4/4L. In the case of $16 \mathrm{~S}$ rRNA the RNA interference against hmtPAP does not seem to have any effect on the steady-state level, while the respective levels of ATP6/8 and CO3 


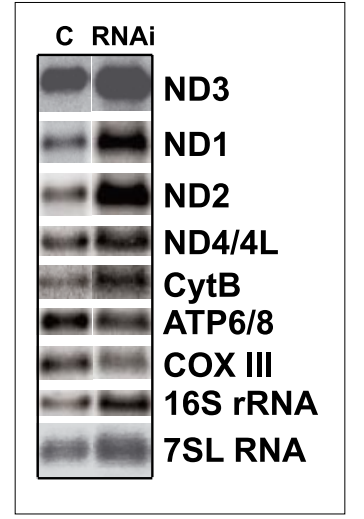

Figure 4. Analysis of the steady-state levels of several mitochondrial RNAs in control and hmtPAP-inhibited HeLa cells.

Seventy two hours after RNAi-mediated silencing RNA samples were isolated from mock-transfected cells (lane C) or cells treated with siRNAs against hmtPAP (lane RNAi) and subjected to the Northern-blot analysis. The same membrane was first hybridized with ND3 probe, and then successively stripped and re-probed with the probes specific for other mtRNAs. 7SL RNA was applied as a loading control.

transcripts (processed out of the same stable precursor - RNA 14/15) tend to be decreased. These experiments strongly suggest that effect of the hmtPAP knock down is transcript-dependent.

\section{DISCUSSION}

Differentiated decay regulates the steady-state levels of the mitochondrial transcripts

The mechanism of transcription of the mtDNA $\mathrm{H}$-strand ensures the same rate of synthesis of each

Table 5. Results of quantitative analysis of steady-state levels for various mitochondrial transcripts in HeLa cells with siRNA-inhibited hmtPAP expression.

The relative steady-state levels of the mtRNAs for the cells treated with siRNAs against hmtPAP were quantified using ImageQuant software. Results are presented as a mean value (fold change comparing to the untreated cells) \pm standard deviation. Only transcripts for which at least three independent experiments had been performed, were taken into account.

\begin{tabular}{lc}
\hline Transcript & Relative steady-state level (vs untreated cells) \\
\hline ND3 & $132 \pm 3$ \\
ND1 & $157 \pm 25$ \\
ND2 & $149 \pm 43$ \\
CO3 & $94 \pm 36$ \\
ATP6/8 & $96 \pm 38$ \\
16S rRNA & $103 \pm 22$ \\
\hline
\end{tabular}

mRNA transcript. Thus, the differentiated steadystate levels of the individual transcripts are achieved by the efficiency of their excision from the long precursor and degradation rate. The rates of synthesis of mitochondrial mRNA species in HeLa cells were analyzed by Gelfand and Attardi (1981) and found to be between 1.5 and 2.1 molecules/min per cell. Lower values obtained for COI and ND4/4L (1.1 and 0.7 molecules/min per cell, respectively) were suggested to be the result of greater degradation of larger molecules during preparation of the RNA samples. Despite the same rate of synthesis, we observed 8-fold difference in the steady-state levels of the mitochondrial transcripts. However, after analysis of 8 of the 10 mRNA transcripts encoded by the $\mathrm{H}$ strand we found that the steady-state levels of the analyzed mRNAs are directly correlated with their decay rates. Five analyzed transcripts (ND1, ND2, CO1, ND5 and Cyt. b) possessed similar steady-state levels and half-lives. We hypothesize that lowered steady-state level of the ND3 transcript and its shorter half-life can be explained by enhanced degradation of defective transcripts occurring during the excision from the precursor. Two transcripts $(\mathrm{CO} 2$ and ATP 6/8) were found to be significantly more stable. A possible explanation is that these mRNAs are stabilized by some secondary structures or interactions with proteins. Remarkedly reduced half-lives in the presence of the ethidium bromide (half-life of $108 \pm$ 2 min for ATP6/8 and $124 \pm 25 \mathrm{~min}$ for $\mathrm{CO} 2$, data not shown) confirm this hypothesis, since ethidium bromide intercalating into RNA may destroy the secondary structures or RNA-protein interactions. The obtained results indicate that the decay rates of the mitochondrial polyadenylated mRNAs are differentiated and are an important factor regulating their steady-state levels.

We also demonstrate that thiamphenicol stabilizes three mitochondrial transcripts (ND2, ND3 and Cyt. b). Translation-dependent turnover of the ND3 transcript can be partially explained by the presence of the ND3 transcripts without a STOP codon. Such aberrant molecules were shown to be rapidly degraded in a translation-dependent pathway (Temperley et al., 2003). However, the STOP codons were absent only in $13 \%$ of the polyadenylated transcripts from cells treated with thiamphenicol and in $21 \%$ of the oligoadenylated transcripts from normal cells. The rather low level of defective mRNA molecules cannot entirely explain the significant stabilization of the ND3 transcript after inhibition of mitochondrial translation. The reason for stabilization two other transcripts is also unknown. Their steadystate levels and half-lives in normal cells do not suggest enhanced degradation. However, cells, in which the half-lives were analyzed, were pre-treated with thiamphenicol for three days. Three-day inhibition 
of the mitochondrial protein synthesis causes a decrease in the activity of respiratory chain complexes. For example, from our other experiments we know that activity of the cytochrome $c$ oxidase in HeLa cells is reduced to $35 \%$ and $16 \%$ after respectively 2 and 4-day treatment with thiamphenicol (unpublished). Thus, it is possible that decreased activity of the respiratory chain or lack of the novel synthesized proteins activate some RNA-binding proteins which increase the stability of the transcripts. A similar phenomenon was observed for the $\mathrm{CO} 2$ transcript after inhibition of the cytochrome $c$ oxidase by sodium azide (Leary et al., 1998).

Inaccurate processing of the mitochondrial $\mathrm{H}$-strand precursor

The first proof of inaccurate processing of the RNA precursor was reported by Dubin et al. (1982) for the $3^{\prime}$ end of the $12 \mathrm{~S}$ rRNA transcript. Here we report that inaccurate processing occurs also at the $3^{\prime}$ end of the ND3 transcripts. Only the first nucleotide of the ND3 STOP codon in encoded by the mtDNA. The full UAA STOP codon is created by the polyadenylation process. The presence of the ND3 transcripts lacking the $U$ nucleotide may indicate that inaccurate processing occurs on the border of the ND3 3' end and tRNA ${ }^{\text {Arg }} 5^{\prime}$ end (Fig. 5). Cleavage in the position -1 from the $3^{\prime}$ end of the ND3 transcript causes the formation of the ND3 mRNAs without STOP codons. Thus, the translation-dependent degradation in untreated cells serves to remove aberrant transcripts formed during the excision of mRNA transcripts from the RNA precursors. Thus, the translation-degradation pathway discovered by Temperley et al. (2003) is a part of the system controlling the quality of the mitochondrial mRNAs.

\section{Origin and destination of the ND3 oligoadenylated transcripts}

The existence of inaccurate processing could suggest that the oligoadenylated transcripts originate

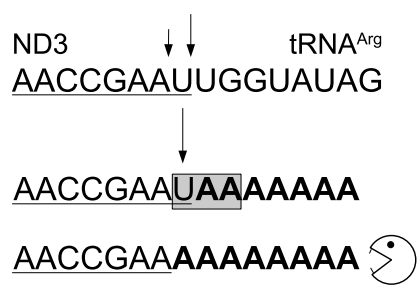

Figure 5. Hypothesis of inaccurate processing of the mitochondrial $\mathrm{H}$-strand precursor.

Inaccurate cleavage of the precursor between $3^{\prime}$ end of the ND3 and the $5^{\prime}$ end of the tRNA ${ }^{\text {Arg }}$ leads to occurrence of the ND3 transcripts lacking the last U nucleotide. Polyadenylation of these aberrant transcripts does not create the UAA STOP codon. from translation-dependent deadenylation. However, our results do not confirm this hypothesis. First of all, the steady-state level of the oligo(A) fraction is not affected by the thiamphenicol treatment. Secondly, despite the presence of the transcripts without STOP codon, the oligo(A) fraction is mainly composed of transcripts with normal 3' ends. Our experiments with actinomycin D indicate that the oligoadenylated ND3 mRNAs are directly linked to the transcription. Data obtained from the sequencing of the ND3 3' ends also indicate that these transcripts originate rather from adenylation of the precursors than deadenylation of defective transcripts.

The destination of the oligoadenylated $\mathrm{mR}$ NAs observed in normal cells is unknown. Its metabolism is relatively fast, since the oligo(A) fraction completely disappears one hour after inhibition of mitochondrial transcription, this could be due to degradation or polyadenylation. However, our analysis of ND3 transcript decay in cells with disrupted poly(A)-polymerase indicates that oligoadenylated mRNAs may undergo polyadenylation to normal transcripts. The last observation suggests that oligoadenylated ND3 transcripts are rather intermediates between the excised naked transcripts and mature fully polyadenylated ones than products of degradation.

The step in which an oligo(A) tail is added immediately follows the excision of the transcript from the precursor. It is worth noting that the reaction must be fast as no "naked" precursors are observed. At least one added adenine was present in each of all 52 sequenced cDNAs belonging to the oligoadenylated fraction (19 from normal cells and 33 after poly(A) polymerase disruption) (Tomecki et al., 2004, and this work). This result suggests a high affinity of the polyadenylating system for nonpolyadenylated precursors and a subsequent decrease of this affinity after addition of a short stretch of adenines. Otherwise two fractions would be observed in cells partially depleted for hmtPAP: one consisting of fully polyadenylated transcripts and a second one of transcripts "waiting" for polyadenylation. Another possible explanation is that another unknown enzyme is responsible for adding the oligo(A) tails and the already identified hmtPAP is responsible only for elongation of the oligo(A) tails to poly(A) tails.

\section{Presence of the poly(A) tail and the stability of the} mitochondrial transcripts

It is striking that in all RNA interference experiments that have been performed to date the steady state-level of ND3 mRNA is significantly elevated following hmtPAP silencing. Careful analysis of the data reported by Nagaike et al. (2005) confirms this statement, when loading control is taken into ac- 
count. We also show that the levels of the other transcripts coding for subunit of complex I (ND1, ND2, ND4/4L) are also elevated. Results presented in this work indicate that the stability of the ND3 transcript is not changed following the hmtPAP knock down. Therefore, the elevated level of the ND3, as well as of other NADH dehydrogenase, is most likely due to activation of the mitochondrial transcription.

Decreased levels of the other mitochondrial transcripts in relation to the transcripts coding for $\mathrm{NADH}$ dehydrogenase subunits suggest their decreased stability, which is observed both by us and Nagaike et al. (2005). Decreased steady-state levels of other mitochondrial transcripts, especially $\mathrm{CO} 2$ and ATP6/8, would be the result of their destabilisation caused by lack of interactions with some RNA-binding proteins, which may bind to the poly(A) tails. We speculate the presence of such proteins for $\mathrm{CO} 2$ and ATP6/8 on the basis of their significant stability in normal cells, which is severely affected in the presence of ethidium bromide.

Taking the results of the quantification summarized in Table 5 into consideration, variation in the relative steady-state levels for some analyzed transcripts is quite high and reflects the variability of the effect of hmtPAP silencing on the steady-state levels that we observe for some mtRNAs between independent experiments. Probably the best example illustrating this problem is the ATP6/8 transcript, for which the steady-state level was found to be evidently increased in our previous study, but is decreased in the particular experiment described in this report and shown in Fig. 4.

\section{CONCLUSIONS}

The varied steady-state levels of the mitochondrial mRNA transcripts are the result of the differentiated stability of the mRNA molecules. Three transcripts (ND2, ND3 and Cyt. b) appeared to be stabilized by treatment with thiamphenicol, which is a known inhibitor of the mitochondrial protein synthesis. Among eight analysed mitochondrial transcripts ND3 was the one with the shortest halflife. Despite that, the stability of the ND3 transcript was not affected by deficient polyadenylation of the mRNA molecules. Analysis of the $3^{\prime}$ ends of the ND3 mRNAs revealed inaccurate processing of the $\mathrm{H}$ strand RNA precursor between ND3 and tRNA ${ }^{A r g}$ locus leading to occasional occurrence of mRNAs without the STOP codon. However, our results suggest that the oligo(A) fraction observed in case of the ND3 transcript is linked rather to ongoing transcription than translation-dependent deadenylation proposed by Temperley et al. (2003) for the ATP6/8 transcripts lacking the STOP codon.

\section{Acknowledgements}

This study was supported by State Committee for Scientific Research (KBN, Poland) Grants 2 P05A 017 26, 2 P04A 00229 and PBZ-KBN 091/P05/2003; Centre of Excellence for Multi-scale Biomolecular Modelling, Bioinformatics and Applications (Poland) and by the Faculty of Biology, Warsaw University Intramural grant BW1601-43. R.T. is the recipient of a scholarship from the Postgraduate School of Molecular Medicine affiliated with the Medical University of Warsaw.

\section{REFERENCES}

Chrzanowska-Lightowlers ZM, Preiss T, Lightowlers RN (1994) Inhibition of mitochondrial protein synthesis promotes increased stability of nuclear-encoded respiratory gene transcripts. J Biol Chem 269: 27322-27328.

Dubin DT, Montoya J, Timko KD, Attardi G (1982) Sequence analysis and precise mapping of the $3^{\prime}$ ends of HeLa cell mitochondrial ribosomal RNAs. J Mol Biol 157: 1-19.

Frischmeyer PA, van Hoof A, O'Donnell K, Guerrerio AL, Parker R, Dietz HC (2002) An mRNA surveillance mechanism that eliminates transcripts lacking termination codons. Science 295: 2258-2261.

Gagliardi D, Leaver CJ (1999) Polyadenylation accelerates the degradation of the mitochondrial mRNA associated with cytoplasmic male sterility in sunflower. EMBO J 18: 3757-3766.

Gelfand R, Attardi G (1981) Synthesis and turnover of mitochondrial ribonucleic acid in HeLa cells: the matureribosomal and messenger ribonucleic acid species are metabolically unstable. Mol Cell Biol 1: 497-511.

Hayes R, Kudla J, Gruissem W (1999) Degrading chloroplast mRNA: the role of polyadenylation. Trends Biochem Sci 24: 199-202.

Jesina P, Tesarova M, Fornuskova D, Vojtiskova A, Pecina P, Kaplanova V, Hansikova H, Zeman J, Houstek J (2004) Diminished synthesis of subunit a (ATP6) and altered function of ATP synthase and cytochrome $c$ oxidase due to the mtDNA $2 \mathrm{bp}$ microdeletion of TA at positions 9205 and 9206. Biochem J 383: 561-571.

LaCava J, Houseley J, Saveanu C, Petfalski E, Thompson E, Jacquier A, Tollervey D (2005) RNA degradation by the exosome is promoted by a nuclear polyadenylation complex. Cell 121: 713-724.

Leary SC, Battersby BJ, Hansford RG, Moyes CD (1998) Interactions between bioenergetics and mitochondrial biogenesis. Biochim Biophys Acta 1365: 522-530.

Litt M, Hauge X, Sharma V (1993) Shadow bands seen when typing polymorphic dinucleotide repeats: some causes and cures. Biotechniques 15: 280-284.

Mitchell P, Tollervey D (2000) mRNA stability in eukaryotes. Curr Opin Genet Dev 10: 193-198.

Nagaike T, Suzuki T, Katoh T, Ueda T (2005) Human mitochondrial mRNAs are stabilized with polyadenylation regulated by mitochondria-specific poly(A) polymerase and polynucleotide phosphorylase. J Biol Chem 280: 19721-19727.

Ojala D, Montoya J, Attardi G (1981) tRNA punctuation model of RNA processing in human mitochondria. $\mathrm{Na}$ ture 290: 470-474. 
Perlman S, Abelson HT, Penman S (1973) Mitochondrial protein synthesis: RNA with the properties of Eukaryotic messenger RNA. Proc Natl Acad Sci USA 70: 350-353.

Sambrook J, Russel DW (2001) Molecular Cloning. A Laboratory Manual 3rd edn, Cold Spring Harbor Laboratory Press, Cold Spring Harbor, New York.

Taanman JW (1999) The mitochondrial genome: structure, transcription, translation and replication. Biochim Biophys Acta 1410: 103-123.

Temperley RJ, Seneca SH, Tonska K, Bartnik E, Bindoff LA, Lightowlers RN, Chrzanowska-Lightowlers ZM (2003) Investigation of a pathogenic mtDNA microdeletion reveals a translation-dependent deadenylation decay pathway in human mitochondria. Hum Mol Genet 12: 2341-2348.
Tomecki R, Dmochowska A, Gewartowski K, Dziembowski A, Stepien PP (2004) Identification of a novel human nuclear-encoded mitochondrial poly(A) polymerase. Nucleic Acids Res 32: 6001-6014.

van Hoof A, Frischmeyer PA, Dietz HC, Parker R (2002) Exosome-mediated recognition and degradation of mRNAs lacking a termination codon. Science 295: 22622264.

Vandesompele J, De Preter K, Pattyn F, Poppe B, Van Roy N, De Paepe A, Speleman F (2002) Accurate normalization of real-time quantitative RT-PCR data by geometric averaging of multiple internal control genes. Genome Biol 3: research 1-0034.11.

Xu F, Cohen SN (1995) RNA degradation in Escherichia coli regulated by $3^{\prime}$ adenylation and $5^{\prime}$ phosphorylation. Nature 374: 180-183. 